\title{
A New Grounded Current Controlled Inductor Based on Simplified Current Conveyors
}

\author{
Zineb M'harzi, Mustapha Alami, and Farid Temcamani
}

\begin{abstract}
In this paper, a new active grounded inductor controlled in current is described. This structure is realized using negative second generation current controlled conveyors and a single grounded capacitor, with no external resistance. The proposed circuit offers many advantages, such as: operation at high frequencies, simple circuit, tuning by the bias current, low power dissipation, etc. Comparison between this topology and those presented in literature is done to highlight the benefits of our structure. As an application, a bandpass filter based on the proposed active inductance is constructed to confirm the usability of the circuit and illustrate these performances. The filter center frequency and quality factor can be tuned independently. Simulation results, given under PSPICE software, present good agreement with the theoretical ones.
\end{abstract}

Keywords-Active inductor, bandpass filter, BiCMOS technology, Integrated Circuit (IC), Second Generation Current Controlled Conveyor (CCCII).

\section{INTRODUCTION}

$\mathbf{T}$ HE electronic devices integration constitutes a permanent challenge in order to reduce the size and the cost, and to increase the frequency capability. Impressive progress was obtain these last decades, particularly to integrate active devices as bipolar or CMOS circuits. Passive elements such as inductors [1] still constitute a real problem to an efficient integration. The inductor is widely used in the implementation of several analog circuits for various applications such as oscillators, active filters, analog phase shifters and cancellation of parasitic inductances [1]-[2]. However, the inductor used in their conventional manufacturing technology presents many problems [1]-[4]:

- It occupies a large chip,

- It includes more parasitic elements,

- It cannot operate at very low and very high frequencies,

- It spreads magnetic energy,

- It does not allow an electronic tuning.

On the other hand, the current mode circuits have known a considerable attention for their interesting advantages. Compared to classical voltage circuits, they have a wider bandwidth, a lower power dissipated, a simpler topology, a higher linearity, etc [2], [5]. Particularly, several inductance simulators operating in current mode have been implemented [1][20]. These structures are based on second generation current conveyors [2], [12]-[15].

The main objective of this paper is to present a new active grounded inductor controlled in current. The novel structure

Z. M'harzi and M. Alami are with the STRS Laboratory, INPT, Rabat, MOROCCO, (e-mail: mharzi@inpt.ac.ma).

F. Temcamani is with the QUARTZ Laboratory, ENSEA, Cergy-Pontoise Cedex, FRANCE. uses a minimal number of active and passive elements, which make it a suitable circuit for IC implementation. Furthermore, this circuit has several advantages compared to other circuits proposed in the literature, and the inductance value can be tuned electronically by changing the bias current.

The organization of this paper is as follows. Section II gives an overview of a simplified negative second generation current controlled conveyor $\left(\mathrm{CCCII}^{-}\right)$used in this work. Section III presents the inductor structure and the theoretical study. In Section IV, a second order bandpass filter which is a simple application of this inductance is described. PSPICE simulations, performed using the parameters of $0.35 \mu \mathrm{m}$ BiCMOS technology of ST [21], are included in Section V.

\section{CURrent CONTRolled CONVEyor}

The second generation current controlled conveyor with negative transfer of current $\left(\mathrm{CCCII}^{-}\right)$used in this paper was introduced recently [22], [23].

The $\mathrm{CCCII}^{-}$equivalent model comprises essentially three inputs-outputs terminals denoted $\mathrm{X}, \mathrm{Y}$, and $\mathrm{Z}$ referenced to the ground. A fourth terminal is relative to the bias current $I_{0}$ (Fig. 1).

The conveyor electrical schematic is presented in Fig. 2. This circuit is implemented from two NPN transistors and four DC current sources. These sources will be, in the real circuit replaced by CMOS current mirrors.

The $\mathrm{CCCII}^{-}$current-voltage matrix relationship is:

$$
\left[\begin{array}{c}
i_{Y} \\
v_{X} \\
i_{Z}
\end{array}\right]=\left[\begin{array}{ccc}
1 / Z_{Y} & 0 & 0 \\
\beta(s) & Z_{X} & 0 \\
0 & -\alpha(s) & 1 / Z_{Z}
\end{array}\right] \cdot\left[\begin{array}{c}
v_{Y} \\
i_{X} \\
v_{Z}
\end{array}\right]
$$

$\beta(s)$ and $\alpha(s)$ are the conveyor voltage and current gains. $Z_{Y}\left(R_{Y} / / C_{Y}\right)$ and $Z_{Z}\left(R_{Z} / / C_{Z}\right)$ are respectively the high input and output impedances at terminals $\mathrm{Y}$ and $\mathrm{Z}$ (ideally infinite). The node $X$ is represented by a low impedance $Z_{X}$ ( $R_{X}$ in series with $L_{X}$ ), where the resistance $R_{X}$ is inversely proportional to the bias current $I_{0}$ according to the equation [23]:

$$
R_{X}=\frac{V_{T}}{I_{0}}
$$

Where, $V_{T}$ is the thermal voltage $\left(\approx 26 \mathrm{mV}\right.$ at $\left.27^{\circ} \mathrm{C}\right)$.

The variation of the parasitic resistance $\mathrm{R}_{X}$ versus the bias current $I_{0}$ is shown in Fig. 3. The values obtained by simulation match well with the theoretical ones.

Moreover, this conveyor is characterized by simple structure, low supply voltage equal to $\pm 0.75 \mathrm{~V}$; and it can be controlled by the bias current $I_{0}$. 


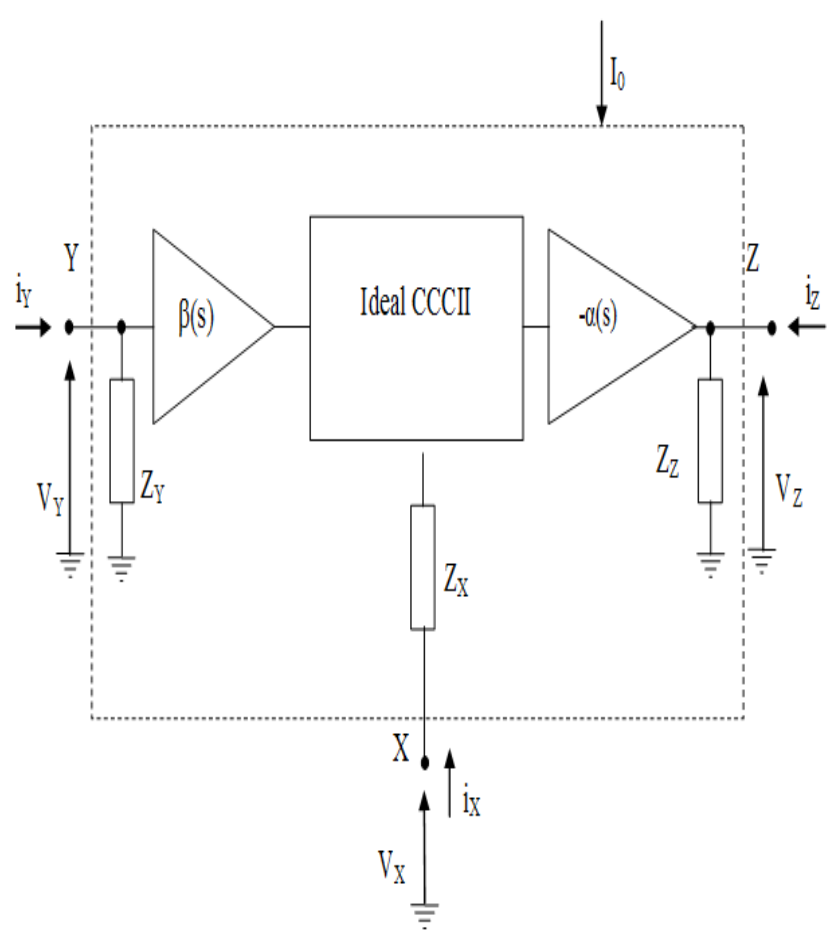

Fig. 1. $\mathrm{CCCII}^{-}$equivalent model with all parasitic elements

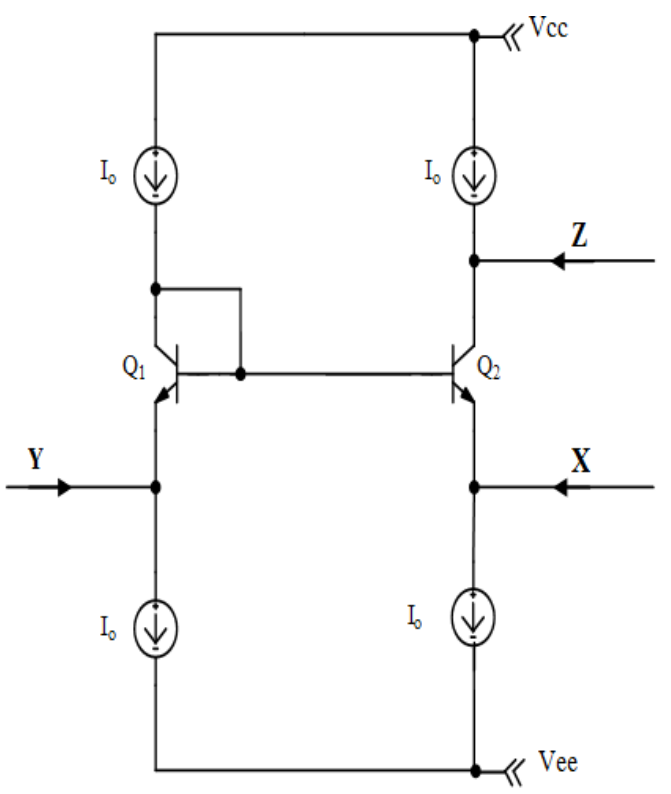

Fig. 2. $\mathrm{CCCII}^{-}$electrical schematic

Furthermore, the minimum number of active elements used leads to a small silicon area, a low power consumption and an excellent frequency behavior in comparison with previews proposed solution, which make it an ideal choice for the tuned analog functions in current and voltage modes [22], [23].

\section{CURRENT CONTROLLED INDUCTOR}

\section{A. Circuit description}

The proposed current controlled inductor is shown in Fig. 4. It consists of two $\mathrm{CCCII}^{-}$conveyors, buffer cells and a

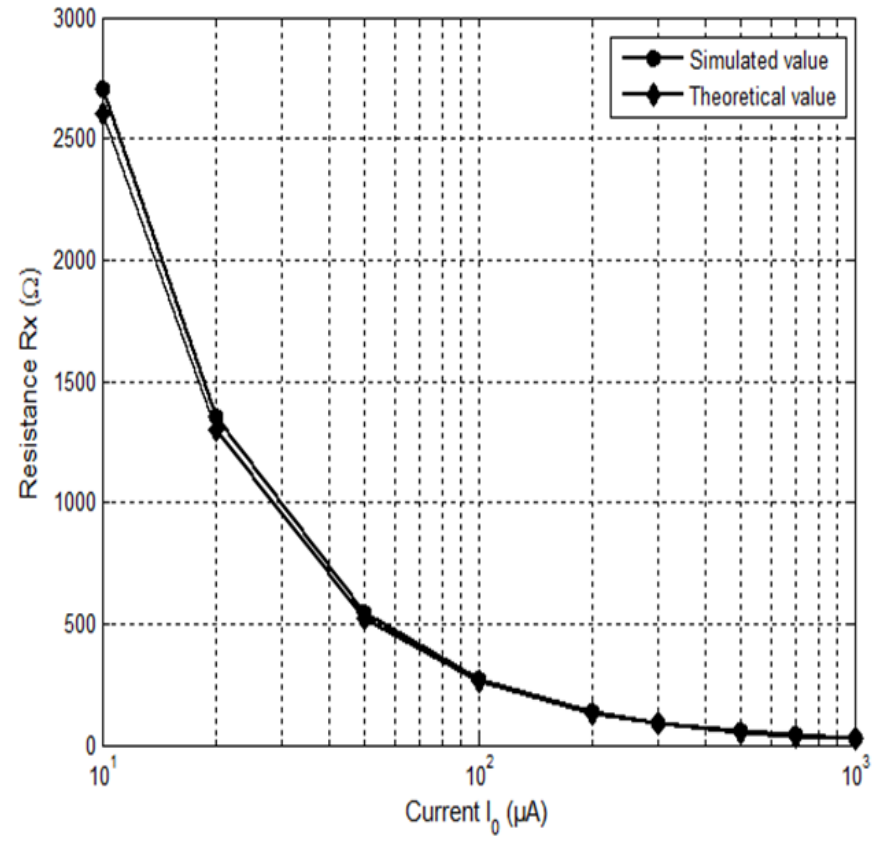

Fig. 3. Variation of resistance $\mathrm{R}_{X}$ versus $\mathrm{I}_{0}$

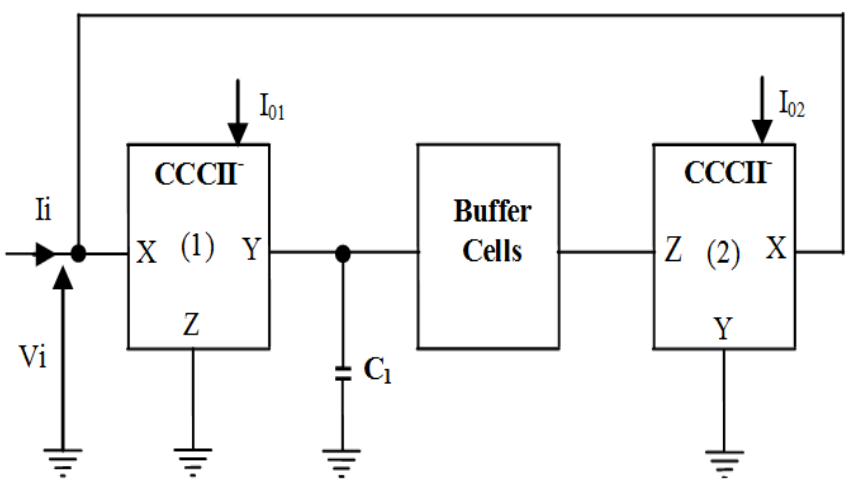

Fig. 4. Current controlled inductance circuit

grounded capacitor. This structure uses no external resistor and does not require any condition of components matching. Moreover, the buffer cells, used in the circuit, are added in order to change the second conveyor sign.

Figure. 5 presents the complete schematic of the inductance circuit. When the both conveyors are supposed ideals, the input impedance of the circuit is expressed as:

$$
Z_{i}=\left(R_{X 1}\right) / /\left(R_{X 2}\right) / /\left(R_{X 1} R_{X 2} j C_{1} \omega\right)
$$

The impedance $Z_{i}$ is equivalent to the active inductance $L_{i}=R_{X 1} R_{X 2} C_{1}$ in parallel with the resistance $R_{i}=R_{X 1} / / R_{X 2}$, where the indexes 1 and 2 refer to the conveyors of Fig. 4.

If the both conveyors have the same bias current $\left(I_{0}=I_{01}=\right.$ $I_{02}$ ) and from (2), the equivalent equation of the inductance becomes:

$$
L_{i}=\left(\frac{V_{T}}{I_{0}}\right)^{2} C_{1}
$$




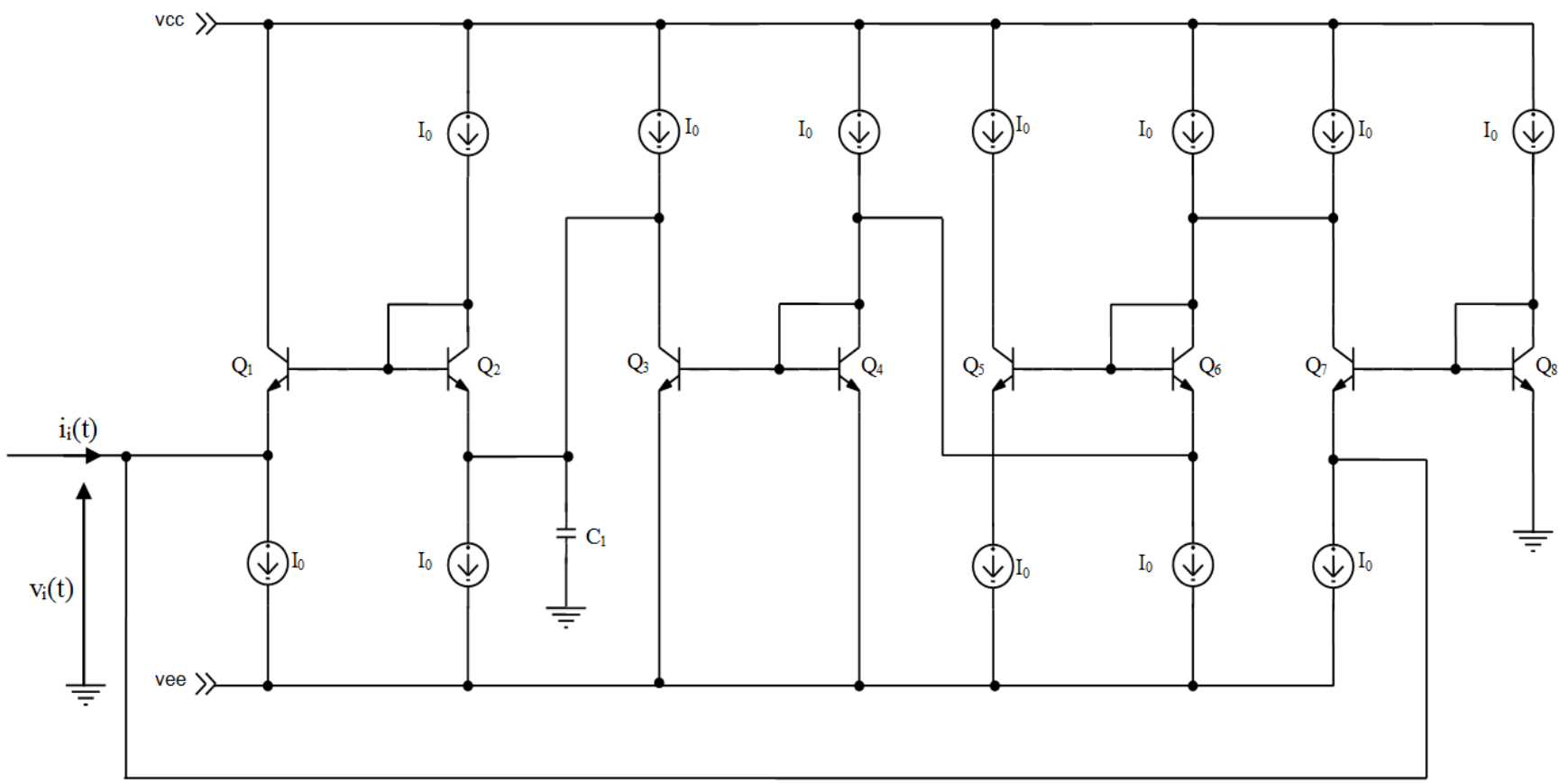

Fig. 5. Current controlled inductance schematic implementation

From this equation, we can clearly conclude that the inductance can be controlled by the bias current of the conveyors $\left(I_{0}\right)$.

\section{B. Non ideality analysis}

Taking into account all the parasitic elements of the conveyors (Fig. 1), the non-ideal input impedance of the inductor circuit (Fig. 4) becomes, where $s=j \omega$ :

$$
Z_{i}=\left(R_{X 1}+L_{X 1} s\right) / /\left(R_{X 2}+L_{X 2} s\right) / / Z_{e q}
$$

With:

$$
Z_{e q}=\frac{\left(R_{X 1}+L_{X 1} s\right) / /\left(R_{X 2}+L_{X 2} s\right)}{\beta_{1}(s) \alpha_{2}(s)} \cdot \frac{1+R_{P} C_{P} s}{R_{P}}
$$

The $R_{P}\left(R_{Y 1} / / R_{Z 2}\right)$ and $C_{P}\left(C_{Y 1}+C_{Z 2}+C_{1}\right)$ are respectively the equivalent parasitics resistor and capacitor of the conveyors at $Y_{1}$ and $Z_{2}$, which appear in parallel with the capacitor $C_{1}$.

Moreover, the impedance $Z_{e q}$ is composed of the inductance $L_{i}$ in series with a parasitic resistance $r_{s}$ :

$$
r_{s}=\frac{R_{X 1} R_{X 2}}{R_{P}}
$$

Figure 6 shows the equivalent diagram of the impedance $Z_{i}$, taking into account the parasitic elements of the conveyors.

\section{Comparison with literature}

The proposed grounded simulated inductor is compared with previously published structures.

The majority of the recent inductor presented in the literature [2]-[6], [8], [10], [14]-[20] are electronically voltage or current tunable, and do not require matching conditions.

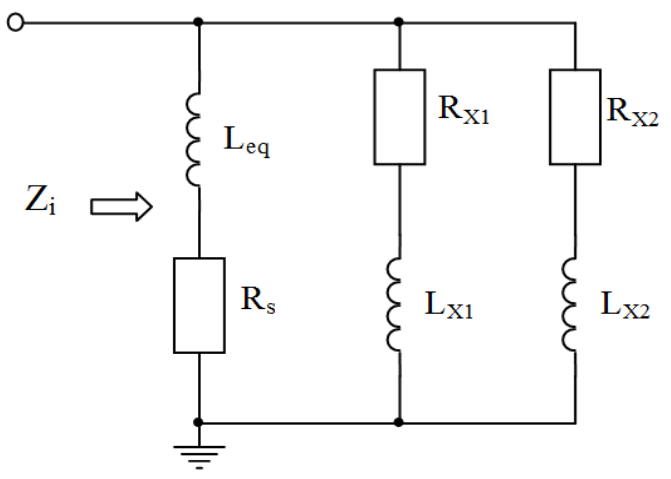

Fig. 6. Equivalent diagram of $Z_{i}$

Moreover, some of them use commercially available devices such as AD844 [2], [6], [19], and the others use other active components, like:

- Second generation current conveyors (CCIIs) [2], [14], [15],

- Fully differential second generation current conveyor (FDCCII) [4],

- Third generation current conveyor (CCIII) [5],

- Negative-type modified inverting current conveyors $\left(\mathrm{MICCI}^{-}{ }^{\mathrm{s}}, \mathrm{MICCII}^{-} \mathrm{s}\right)$ [3], [20],

- Current feedback operational amplifier (CFOA) [6], [18],

- Voltage differencing differential input buffered amplifiers (VD-DIBA) [8],

- Dual X second generation current conveyor (DXCCII) [16], [19],

- Current Amplifier [17], 


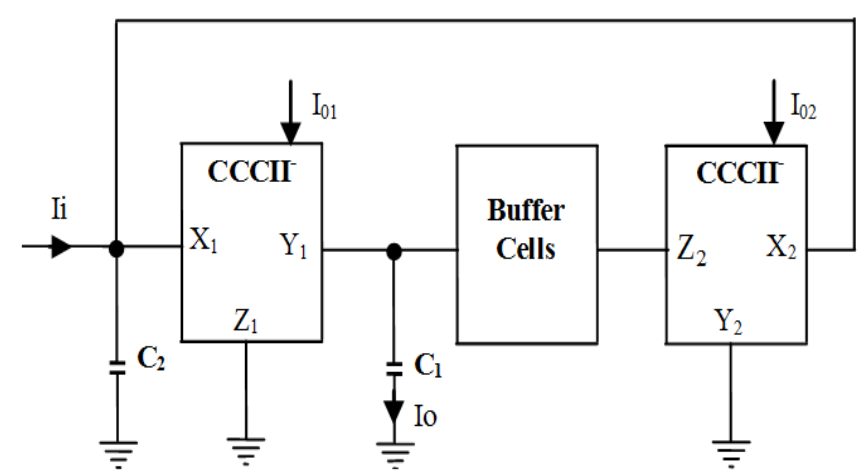

Fig. 7. Current mode second order bandpass filter

- Current differencing transconductance amplifiers (CDTAs) [11].

Nevertheless, these structures suffer from many problems:

- Use of a large number of passive elements [2]-[6], [14], [16], [18]-[20],

- Use of many active elements which affect the power consumption and the area of the circuits [4], [8], [10], [14], [16], [17], [20],

- Use of floating passive components which is not suitable for the integrated circuit implementation point of view [3], [5], [6], [14], [16], [18]-[20],

- Use of PNP transistors which affect the circuit frequency performance [5], [15].

Whereas, the proposed grounded inductance has much more advantages as these structures presented in literature. It has a simpler structure thanks to the simplified current controlled conveyor topology. It uses only a single grounded capacitor with no floating components, which allows it to have a small area, low power consumption and a high frequency performance. In addition, our structure can be controlled in current With these features, the proposed inductor is an attractive element for many applications.

\section{SECOND ORDER BANDPASS FILTER}

To evaluate the performance of the proposed inductance, a current mode second order bandpass filter is presented (Fig. 7).

The filter transfer function is given by:

$$
H(s)=\frac{I_{o}}{I_{i}}(s)=\frac{-R_{X 1} C_{1} s}{1+\left(R_{X 1}+R_{X 2}\right) C_{1} s+R_{X 1} R_{X 2} C_{1} C_{2} s^{2}}
$$

The filter characteristic parameters can be written as:

$$
\begin{gathered}
\omega_{0}=\sqrt{\frac{1}{R_{X 1} R_{X 2} C_{1} C_{2}}} \\
Q=\frac{\sqrt{R_{X 1} R_{X 2}}}{R_{X 1}+R_{X 2}} \cdot \sqrt{\frac{C_{2}}{C_{1}}} \\
H_{0}=\frac{R_{X 1}}{R_{X 1}+R_{X 2}}
\end{gathered}
$$

Where: $f_{0}=\omega_{0} / 2 \Pi$ is the filter center frequency, $Q$ denote the quality factor and $H_{0}$ represents the gain.

Note that in calculation, we need to take into account the parasitic capacitances that appear in parallel with $C_{1}$.

When both conveyors are assumed to be identical, we have:

$$
\begin{gathered}
\omega_{0}=\frac{1}{R_{X} \sqrt{C_{1} C_{2}}} \\
Q=\frac{1}{2} \cdot \sqrt{\frac{C_{2}}{C_{1}}} \\
H_{0}=\frac{1}{2}
\end{gathered}
$$

From these equations, we can see that the quality factor $Q$ is independent of the resistance $\mathrm{R}_{X}$, which is inversely propositional to the bias current $\mathrm{I}_{0}$. In opposite, the center frequency $f_{0}$ depends on the resistance $R_{X}$ and can be controlled by varying the current $\mathrm{I}_{0}$. Moreover, the filter center frequency and quality factor can be tuned independently of each other, which means that we can have high $Q$ and $f_{0}$ at the same time.

\section{Simulation RESUlts AND DISCUSSION}

Simulation results of the various circuits presented in this paper have been obtained using PSPICE software with the parameters of $0.35 \mu \mathrm{m}$ BiCMOS technology from ST [21], and the circuits are supplied with $\pm 0.75 \mathrm{~V}$.

\section{A. Current controlled inductor}

The proposed inductor structure is simulated for an equal bias current for the both conveyors $I_{01}=I_{02}=I_{0}=100 \mu \mathrm{A}$ and the capacitance equal to $C_{1}=10 p F$.

The magnitude and the phase of the input impedance are represented in Fig. 8. In this figure, we compare simulated results obtained with the global electrical circuit (Fig. 5), and the ideal results obtained with its equivalent circuit (Fig. 6 ). The both magnitude curves are in perfect agreement for frequencies between $200 \mathrm{KHz}$ and $20 \mathrm{MHz}$, and for the other frequencies the difference is less than $10 \%$. For the phase figure, both curves are almost identical up to frequencies of the order of $20 \mathrm{MHz}$.

In addition, the values of the inductance and the series resistance found by simulation (Fig. 9) are in good agreement with theoretical ones calculated from (4) and (7). They are respectively equal to $0.77 \mu \mathrm{H}$ and $1.5 \Omega$ for simulated values, against $0.74 \mu \mathrm{H}$ and $1.7 \Omega$ for the theoretical ones. Figure 10 shows the theoretical and the simulated evolutions of the inductance as a function of the bias current $I_{0}=I_{01}=I_{02}$. It is clear that the simulated values are well correlated with theoretical ones. Therefore, this result confirms that, the inductance values can be tuned by changing the bias current of the conveyors. Moreover, the power consumption of this circuit is equal to $1.13 \mathrm{~mW}$. 


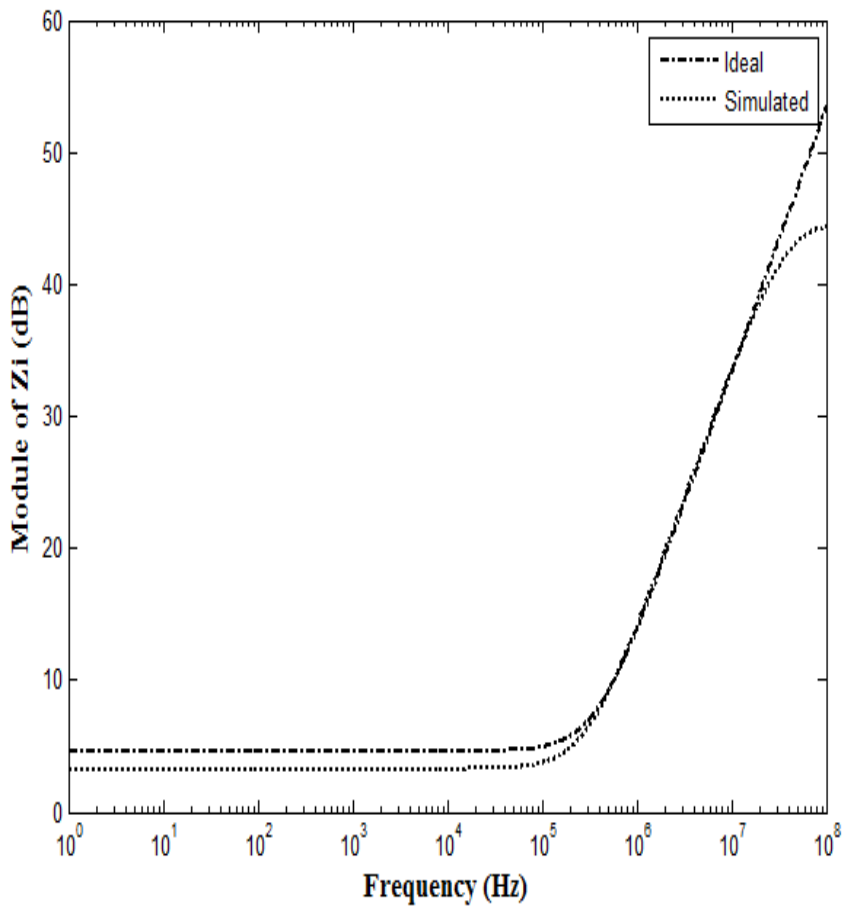

(a) Magnitude

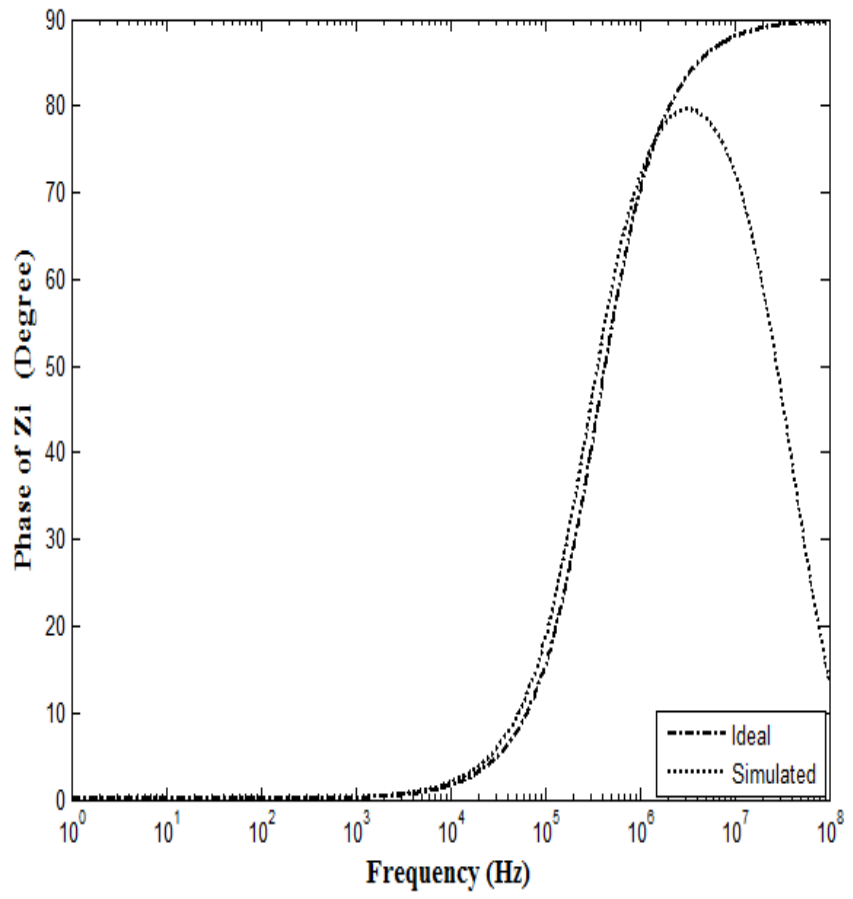

(b) Phase

Fig. 8. Input impedance of the grounded inductor circuit $\begin{array}{ll}\text { (a) Magnitude } & \text { (b) Phase }\end{array}$

TABLE I

FILTER CHARACTERISTICS

\begin{tabular}{|c|c|}
\hline Parameter & Simulated results \\
\hline$f_{0}$ & $17.6 \mathrm{MHz}$ \\
\hline $\mathrm{Q}$ & 2.5 \\
\hline$H_{0}$ & 0.53 \\
\hline Power consumption & $1.13 \mathrm{~mW}$ \\
\hline
\end{tabular}

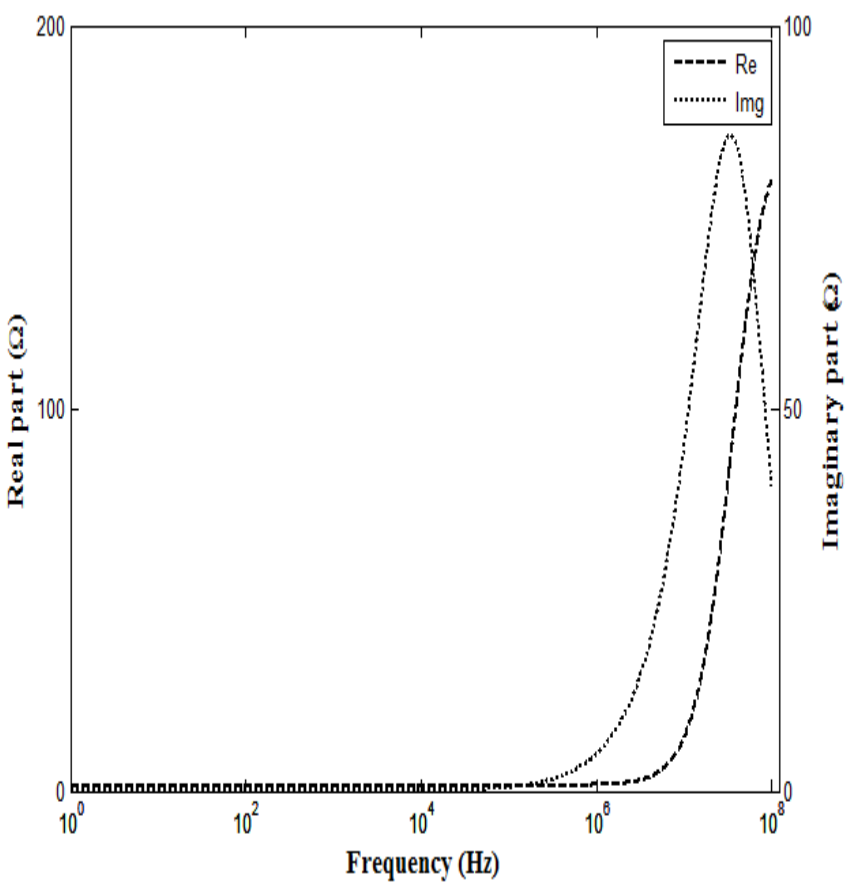

Fig. 9. Real and imaginary parts of the simulated inductance

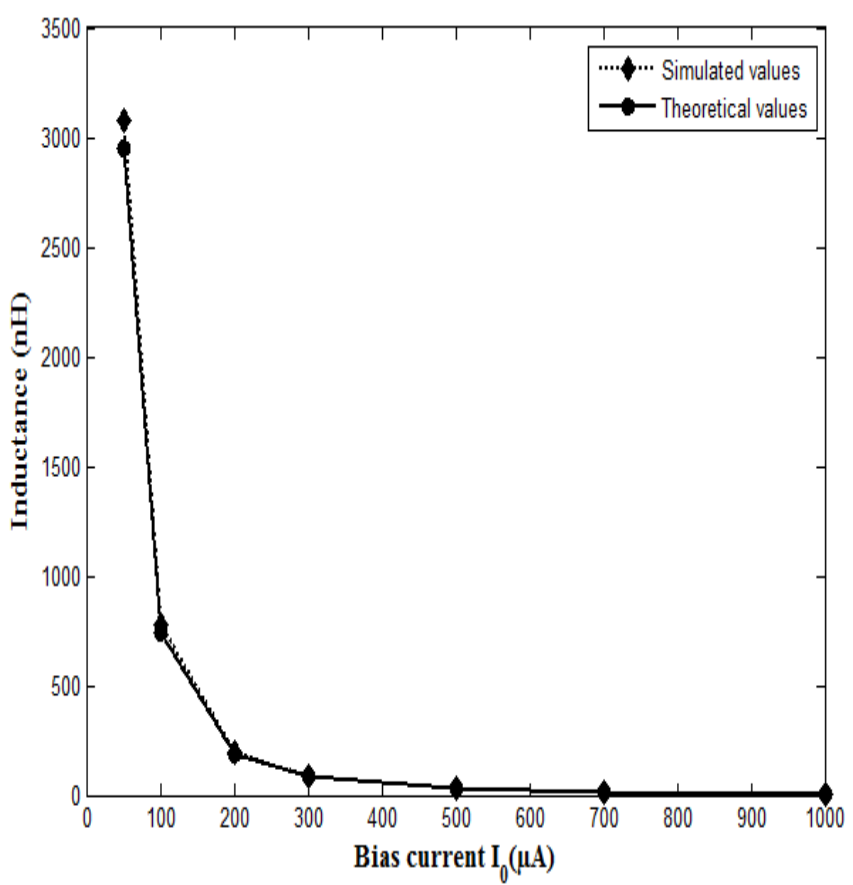

Fig. 10. Evolution of the inductance simulated and theoretical values as a function of bias current $I_{0}=I_{01}=I_{02}$

\section{B. Current mode bandpass filter}

To validate the theoretical analysis, we simulated the second order bandpass filter (Fig. 7) under the following conditions $I_{0}=100 \mu A, C_{1}=8 p F$ and $C_{2}=130 p F$. The main characteristics of this filter are summarized in Table I.

Figure 11 shows the simulated values of the bandpass filter for different values of $I_{0}$. The center frequencies of the filter are $17.6 \mathrm{MHz}, 35 \mathrm{MHz}, 52.24 \mathrm{MHz}$ and $69.4 \mathrm{MHz}$, respectively 


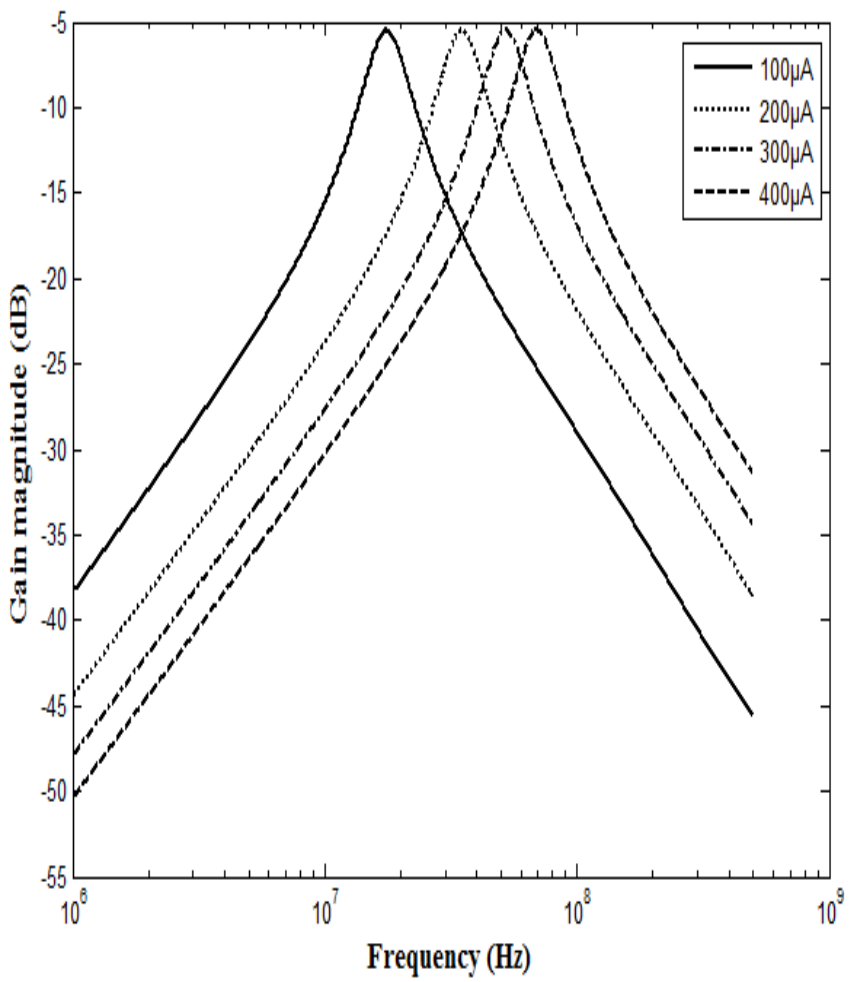

Fig. 11. Frequency response of the filter for different values of $I_{0}$

for $I_{0}$ equal to $100 \mu \mathrm{A}, 200 \mu \mathrm{A}, 300 \mu \mathrm{A}$ and $400 \mu \mathrm{A}$. These values are very close to the theoretical ones $(18 \mathrm{MHz}, 36 \mathrm{MHz}$, $54 \mathrm{MHz}$ and $72 \mathrm{MHz}$, respectively).

Moreover, the simulated quality factors for the same bias currents are about 2.5 , whereas the theoretical values are equal to 2 .

To summarize, we notice that the theoretical and simulated values of the center frequency and the quality factor of the filter are very close; and that, the $f_{0}$ and the $\mathrm{Q}$ can be tuned independently. The variation of the bias current of the conveyors allows the control of the frequency values but not the Q-factor values.

\section{CONCLUSION}

An active grounded inductance simulator controlled in current is presented in this paper. This circuit uses a minimum number of active and passive components. It is composed of two second generation current controlled conveyors and a grounded capacitor. The proposed structure presents several features, such as: simplicity, low supply voltage, low power consumption, possibility of tuning, and use of a single grounded passive component which make it a suitable element for the IC manufacturing. Due to these advantages, the presented inductor is an attractive component for several applications in analog communication systems.

As an application, a current mode second order bandpass filter is realized to illustrate the practical use and the benefits of the proposed inductor structure. This filter is characterized by low power dissipation $(1.13 \mathrm{~mW})$, low supply voltage $( \pm 0.75 \mathrm{~V})$, and the possibility of tuning the frequency independently of the quality factor, which means that we can have a high frequency and a high Q-factor with the same circuit.

\section{REFERENCES}

[1] M. Sagbas, "Component reduced floating $\pm L, \pm C$ and $\pm R$ simulators with grounded passive components," International Journal of Electronics and Communications (AEU), vol. 65, no. 10, pp. 794-798, 2011.

[2] M. O. Çiçekoğlu, "Active simulation of grounded inductors with CCII+s and grounded passive elements," International Journal of Electronics, vol. 85 , no. 4, pp. 455-462, 1998 .

[3] F. Kaçar, "New lossless inductance simulators realization using a minimum active and passive components," Microelectronics Journal, vol. 41, no. 2, pp. 109-113, 2010.

[4] E. Yuce, S. Minaei, and O. Çiçekoğlu, "A novel grounded inductor realization using a minimum number of active and passive components," ETRI Journal, vol. 27, no. 4, pp. 427-432, 2005.

[5] H. Kuntman, M. Gülsoy, and O. Çiçekoğlu, "Actively simulated grounded lossy inductors using third generation current conveyors,' Microelectronics Journal, vol. 31, pp. 245-250, 2000.

[6] E. Yuce, "Novel lossless and lossy grounded inductor simulators consisting of a canonical number of components," Analog Integrated Circuits and Signal Processing, vol. 59, pp. 77-82, 2009.

[7] M. Sagbas, U. E. Ayten, H. Sedef, and M. Koksal, "Electronically tunable floating inductance simulator," International Journal of Electronics and Communications (AEU), vol. 63, no. 5, pp. 423-427, 2009.

8] D. Prasad, D. R. Bhaskar, and K. L. Pushkar, "Realization of new electronically controllable grounded and floating simulated inductance circuits using voltage differencing differential input buffered amplifiers," Active and Passive Electronic Components, 2011.

[9] E. Yuce, and S. Minaei, "A modified CFOA and its applications to simulated inductors, capacitance multipliers, and analog filters," IEEE Transactions on Circuits and Systems I: Regular Papers, vol. 55, no. 1, pp. 266-275, 2008.

[10] D. Prasad, D. R. Bhaskar, and A. K. Singh, "New grounded and floating simulated inductance circuits using current differencing transconductance amplifiers," Radioengineering, vol. 19, no. 1, pp. 194-198, 2010.

[11] A. U. Keskin, and E. Hancioglu, "CDBA-based synthetic floating inductance circuits with electronic tuning properties," ETRI Journal, vol. 27, no. 2, pp. 239-242, 2005.

[12] B. Metin, and O. Cicekoglu, "A novel floating lossy inductance realization topology with NICs using current conveyors," IEEE Transactions On Circuits And Systems II: Express Briefs, vol. 53, no. 6, pp. 483-486, 2006.

[13] PV. A. Mohan, "Grounded capacitor based grounded and floating inductance simulation using current conveyors," Electronics letters, vol. 34, no. 11, pp. 1037-1038, 1998.

[14] E. Yuce, "Grounded inductor simulators with improved low frequency performances," IEEE Transactions on Instrumentation and Measurement, vol. 57, no. 5, pp. 1079-1084, 2008.

[15] A. Fabre, O. Saaid, F. wiest, and C. Boucheron, "Low power current mode second order bandpass IF filter," IEEE Transactions on Circuits and Systems II: Analog and Digital Signal Processing, vol. 44, no. 6, pp. 436-446, 1997.

[16] F. Kaçar, and A. Yeşil, "Novel grounded parallel inductance simulators realization using a minimum number of active and passive components," Microelectronics Journal, vol. 41, no. 10, pp. 632-638, 2010.

[17] C. Psychalinos, and A. Spanidou, "Current amplifier based grounded and floating inductance simulators," International Journal of Electronics and Communications (AEU), vol. 60, no. 2, pp. 168-171, 2006.

[18] F. Kaçar, and H. Kuntman, "CFOA-based lossless and lossy inductance simulators," Radioengineering, vol. 20, no. 3, pp. 627-631, 2011.

[19] I. Myderrizi, S. Minaei, and E. Yuce, "DXCCII-based grounded inductance simulators and filter applications," Microelectronics Journal, vol. 42, no. 9, pp. 1074-1081, 2011.

[20] E. Yuce,"Inductor implementation using a canonical number of active and passive elements," International Journal of Electronics, vol. 94, no. 4, pp. 317-326, 2007.

[21] STMicroelectronics, "0.35 m SiGe BiCMOS", Grenoble (France), 1994

[22] M. Alami, A. Fabre, A. Jaadoud, and A. Touhami, "Simplified BiCMOS current controlled conveyor," in Proc. of 14th IEEE International Conference on Electronics, Circuits and Systems (ICECS), 2007, pp. 383-386.

[23] Z. M harzi, M. Alami, and F. Temcamani, "Improvement of current mode controlled amplifier using current conveyors," in Proc. of WCSIT, 2014, pp. $2-4$ 